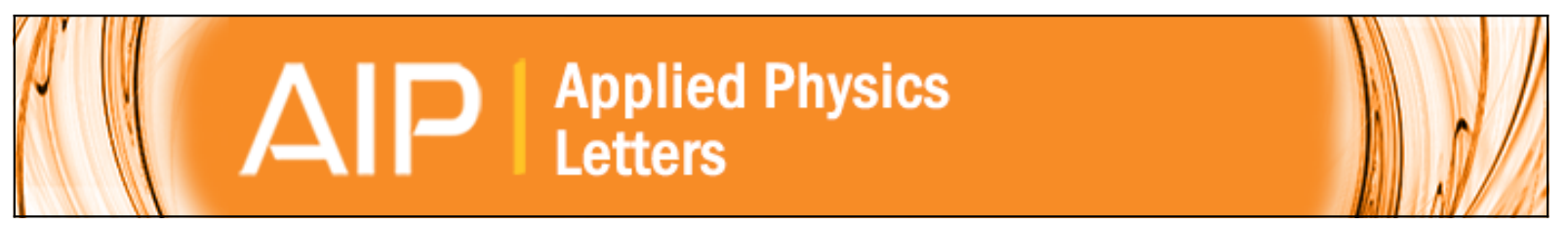

\title{
Towards artifact-free atomic-resolution elemental mapping with electron energy-loss spectroscopy
}

Y. Zhu, A. Soukiassian, D. G. Schlom, D. A. Muller, and C. Dwyer

Citation: Applied Physics Letters 103, 141908 (2013); doi: 10.1063/1.4823704

View online: http://dx.doi.org/10.1063/1.4823704

View Table of Contents: http://scitation.aip.org/content/aip/journal/apl/103/14?ver=pdfcov

Published by the AIP Publishing

\section{Articles you may be interested in}

Visualization of hybridization states with atomic resolution using electron energy loss spectroscopy mapping Appl. Phys. Lett. 100, 163107 (2012); 10.1063/1.4704558

Melting and supercooling studies in submicron Al particles using valence electron energy-loss spectroscopy in a transmission electron microscope

J. Appl. Phys. 110, 024908 (2011); 10.1063/1.3609063

Crystal and electronic structure of lithiated nanosized rutile TiO 2 by electron diffraction and electron energy-loss spectroscopy

Appl. Phys. Lett. 94, 233116 (2009); 10.1063/1.3152783

Atomic-scale characterization of perovskite superlattice using chemical lattice imaging and spatially resolved electron energy-loss spectroscopy

Appl. Phys. Lett. 84, 5374 (2004); 10.1063/1.1767279

Electron energy-loss spectroscopy study of a (LaMnO 3 ) 8 (SrMnO 3 ) 4 heterostructure

Appl. Phys. Lett. 79, 2037 (2001); 10.1063/1.1403316

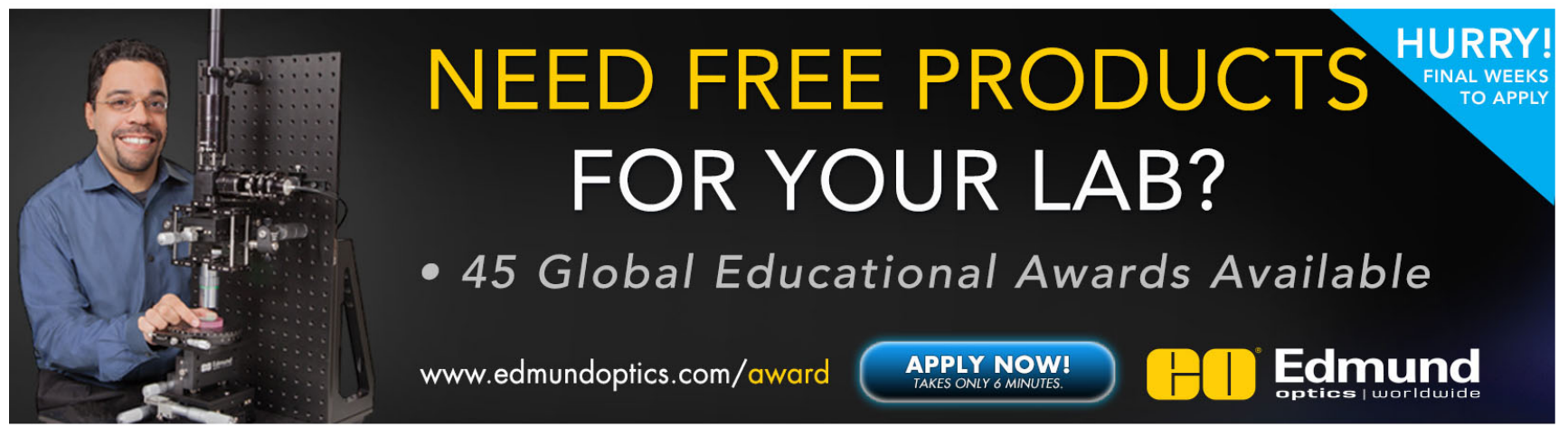




\title{
Towards artifact-free atomic-resolution elemental mapping with electron energy-loss spectroscopy
}

\author{
Y. Zhu, ${ }^{1,2}$ A. Soukiassian, ${ }^{3}$ D. G. Schlom, ${ }^{3,4}$ D. A. Muller, ${ }^{4,5}$ and C. Dwyer ${ }^{1,2,6, a)}$ \\ ${ }^{1}$ Monash Centre for Electron Microscopy, Monash University, Victoria 3800, Australia \\ ${ }^{2}$ Department of Materials Engineering, Monash University, Victoria 3800, Australia \\ ${ }^{3}$ Department of Materials Science and Engineering, Cornell University, Ithaca, New York 14853, USA \\ ${ }^{4}$ Kavli Institute at Cornell for Nanoscale Science, Ithaca, New York 14853, USA \\ ${ }^{5}$ School of Applied and Engineering Physics, Cornell University, Ithaca, New York 14853, USA \\ ${ }^{6}$ Ernst Ruska-Centre for Microscopy and Spectroscopy with Electrons, and Peter Grünberg Institute, \\ Forschungszentrum Jülich, D-52425 Jülich, Germany
}

(Received 10 July 2013; accepted 14 September 2013; published online 1 October 2013)

\begin{abstract}
Atomic-resolution elemental maps of materials obtained using energy-loss spectroscopy in the scanning transmission electron microscope (STEM) can contain artifacts associated with strong elastic scattering of the STEM probe. We demonstrate how recent advances in instrumentation enable a simple and robust approach to reduce such artifacts and produce atomic-resolution elemental maps amenable to direct visual interpretation. The concept is demonstrated experimentally for a $\left(\mathrm{BaTiO}_{3}\right)_{8} /\left(\mathrm{SrTiO}_{3}\right)_{4}$ heterostructure, and simulations are used for quantitative analysis. We also demonstrate that the approach can be used to eliminate the atomic-resolution elastic contrast in maps obtained from lower-energy excitations, such as plasmon excitations. (c) 2013 AIP Publishing LLC. [http://dx.doi.org/10.1063/1.4823704]
\end{abstract}

Over the last decade, atomic-resolution core-level spectroscopy has developed to become an extremely powerful tool for probing nanomaterials. ${ }^{1-8}$ Performed in a scanning transmission electron microscope (STEM), the technique uses an atomic-sized electron probe to excite core-level electrons in the sample, while detectors monitor the spectra of energy-loss electrons and/or the flux of characteristic x-rays. A wealth of atomic-scale information is provided, ${ }^{3,4,9-11}$ including the locations and species of atoms, i.e., elemental maps, and, in the case of electron energy-loss spectroscopy (EELS), information on electronic bonding. While the benefits of using EELS over characteristic $x$-rays include superior detection efficiency, access to bonding information, and the ability to map light elements, atomic-resolution elemental maps acquired using EELS are, in fact, prone to artifacts associated with elastic scattering of the electron probe within the sample. ${ }^{12-14}$ Such artifacts, which occur especially in the presence of heavy elements, can confound data interpretation to the extent that simulations must be invoked to gain a reliable understanding. $\mathrm{X}$-ray elemental maps, on the other hand, are less prone to this effect because any probe scattering following a core-level excitation cannot influence the $\mathrm{x}$-ray yield. ${ }^{7,8}$ In this letter, we demonstrate a simple and robust approach that places the interpretation of EELS elemental maps on essentially the same footing as x-ray elemental maps. Our approach, which applies recent advances in spectrometer instrumentation, uses the simultaneously acquired elastic signal to correct artifacts, often enabling direct visual interpretation of atomic-resolution EELS elemental maps, and thereby simplifying the use of EELS for probing structure and bonding at the atomic scale.

The material used in this study is a superlattice heterostructure $\left[\left(\mathrm{BaTiO}_{3}\right)_{8} /\left(\mathrm{SrTiO}_{3}\right)_{4}\right]_{40}$ grown on a (101) $\mathrm{SmScO}_{3}$ substrate by molecular-beam epitaxy. ${ }^{15,16}$ This material enables us to test our approach in the presence of both strongly

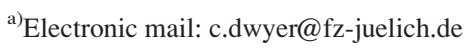

(Sr, Ba) and weakly scattering (O) elements. Samples for observation were prepared by mechanical wedge polishing followed by Ar-ion milling. Atomic-resolution EELS was performed on a Nion $100 \mathrm{kV}$ UltraSTEM, with a beam convergence semi-angle of $30 \mathrm{mrad}$ and an EELS collection semi-angle of $80 \mathrm{mrad}$. The DualEELS mode of a Gatan Enfinium ${ }^{\mathrm{TM}} \mathrm{ER}$ spectrometer was used: Higher energy losses (320-832 eV), including the Ti- $L_{2,3}, \mathrm{O}-K$ and Ba- $M_{4,5}$ edges, were recorded in an acquisition time of $10 \mathrm{~ms}$, while lower losses $(-50-462 \mathrm{eV})$, including the zero-loss peak (ZLP), plasmon peaks, and Ba- $N_{4,5}$ edge, were recorded in $0.5 \mathrm{~ms}$ to prevent detector saturation. Core-loss background subtraction combined power-law fitting and local background averaging. ${ }^{17}$ Annular-dark-field (ADF) images were recorded simultaneously with the EEL spectra using an inner semiangle of $98 \mathrm{mrad}$.

The atomic-resolution ADF image and elemental maps of the heterostructure are shown in Figs. 1(a), 1(e)-1(h). The images are $234 \times 84$ pixels spanning $4 \mathrm{~nm} \times 16 \mathrm{~nm}$, which includes multiple $\left(\mathrm{BaTiO}_{3}\right)_{8}$ and $\left(\mathrm{SrTiO}_{3}\right)_{4}$ layers. In the $\mathrm{ADF}$ image, $\left(\mathrm{BaTiO}_{3}\right)_{8}$ layers show higher intensity than $\left(\mathrm{SrTiO}_{3}\right)_{4}$ layers, consistent with the $Z$-contrast mechanism, and the overall intensity decreases from left to right due to a thickness gradient. Figure 1(d) shows a (spliced) EEL spectrum containing the core-loss edges used for mapping. Individual atomic columns are clearly resolved in both the Ti- $L_{2,3}$ and Ba- $M_{4,5}$ maps. In the $\mathrm{Ba}-M_{4,5}$ map, the middle $\left(\mathrm{BaTiO}_{3}\right)_{8}$ layer contains seven layers of strong Ba columns and two layers of weak Ba columns instead of eight complete layers of $\mathrm{Ba}$. This is due to either interdiffusion of $\mathrm{Ba}$ and $\mathrm{Sr}$ or an interface step originating from the substrate. In the Ba- $N_{4,5}$ map, Ba columns show less contrast due to the greater inelastic delocalization of the Ba- $N_{4,5}$ signal and the lower signal-to-noise ratio (SNR) associated with the $0.5 \mathrm{~ms}$ exposure. On the other hand, the low contrast in the O-K map is due to the small O- $K$ jump ratio in this relatively thick sample. 


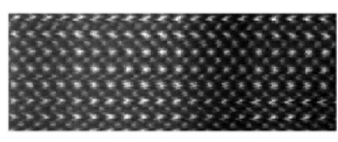

(a)

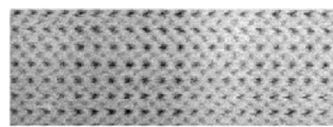

(b)
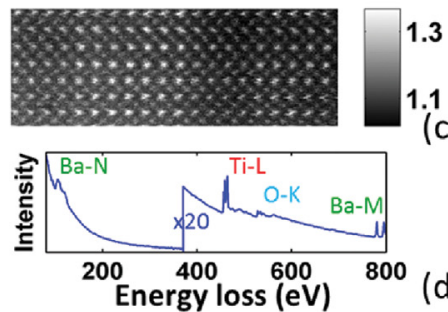

1.3

(c)
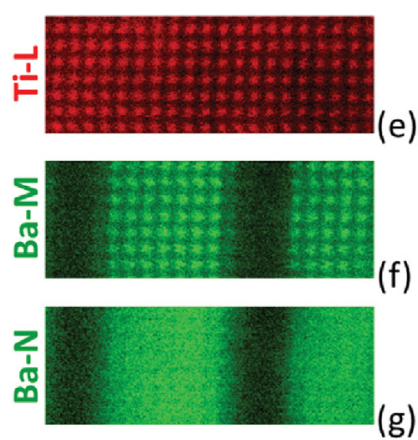

(d)

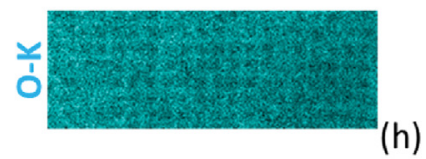

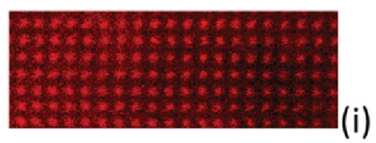
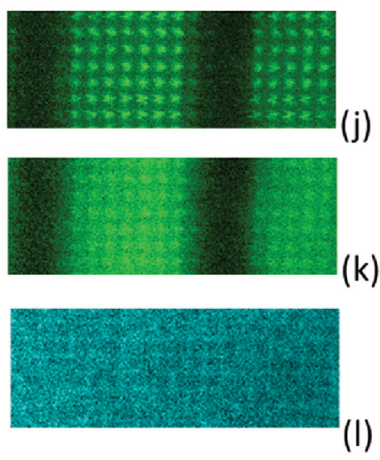
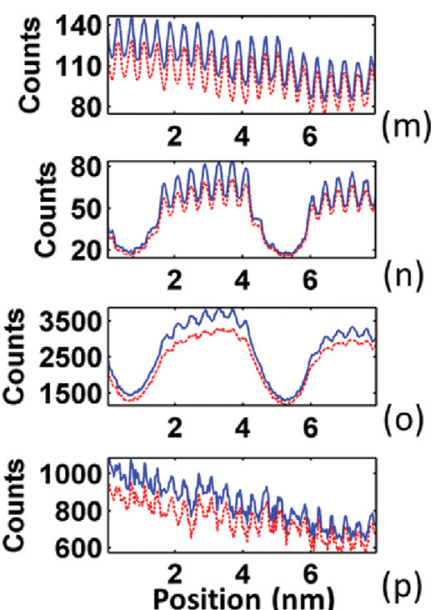

FIG. 1. Atomic-resolution elemental mapping of a $\left(\mathrm{BaTiO}_{3}\right)_{8} /\left(\mathrm{SrTiO}_{3}\right)_{4}$ heterostructure. (a) ADF image; (b) IBF image; (c) elastic-correction map; (d) example EEL spectrum; (e-h) core-loss elemental maps; (i-l) elastic-corrected elemental maps; (m-p) 1D profiles from averaging conventional (dotted lines) and corrected (solid lines) maps over the vertical direction.

As demonstrated by the above results, atomic-resolution STEM-EELS is a powerful tool for characterizing materials. However, it has also been revealed that extra caution is required to properly interpret the contrast of maps acquired at the atomic scale. ${ }^{2,12-14,18-20}$ In particular, a substantial component of undesirable "elastic contrast" can be preserved due to probe electrons being elastically scattered beyond the EELS collection angle. Such large-angle elastic scattering occurs especially in the presence of heavy-atom columns, and it is the basic mechanism to form an incoherent bright-field (IBF) image. If the scattering angles associated with relevant core-level excitations are small compared to the collection angle, as they are here, the contrast in the EELS elemental maps can be interpreted as a multiplication of chemical and elastic (IBF) contrast (an approximation that becomes more accurate for lower core losses). When elastic contrast dominates, which can occur especially for heavy-atom columns, lower energy losses, or small collection angles, artifacts such as volcano-like structures ${ }^{12,13,18,21}$ and contrast reversals on atomic columns $\mathrm{s}^{2,20,22}$ have been reported. On the other hand, these effects are significantly reduced in characteristic $\mathrm{x}$-ray maps because such maps are impervious to any scattering that follows the core-level excitations. ${ }^{7,8}$

To reduce elastic contrast in EELS maps, we apply recent advances in spectrometer instrumentation to record the total intensity falling within the EELS collection angle: Recording the total intensity, including the ZLP, requires a fast acquisition to avoid detector saturation. However, it is not practical to record the ZLP and core-loss edges together in a single fast acquisition because the core-loss SNR will be too low to give good chemical contrast. Recording two different energy regions of the spectra simultaneously with different acquisition times, as described above, solves this issue (here "simultaneous" actually means recording in rapid succession from the same region under the same experimental conditions). The map formed by the total EELS signal is an IBF image (Fig. 1(b)), and for typical sample thicknesses used in STEM it is dominated by elastic and thermal-diffuse scattering. We integrate only the low-loss spectrum at each pixel to form the IBF image because higher losses comprise $<1 \%$ of the total intensity. We then normalize the IBF image using the maximal intensity before taking the reciprocal to obtain a correction map (Fig. 1(c)), which, when used to scale the EELS maps pixel-by-pixel, compensates for the elastic scattering outside of the EELS collection angle. The correction can be applied either before or after generating the elemental maps from the EELS data, giving very similar results in each case.

The corrected elemental maps are shown in Figs. 1(i)-1(l), while Figs. 1(m)-1(p) show 1D profiles obtained by averaging maps along the vertical direction. As evidenced from the correction map, the intensity of heavy-atom columns such as $\mathrm{Ba}$ and $\mathrm{Sr}$ will be enhanced. The contrast of the $\mathrm{Ba}-M_{4,5}$ and $\mathrm{Ba}-N_{4,5}$ maps is significantly improved after correction, with the $\mathrm{Ba}$ columns becoming well-resolved in the corrected $\mathrm{Ba}-N_{4,5}$ map. For the O- $K$ map, which is originally noisy, the contrast is, in fact, lower after correction. This indicates that, despite the large EELS collection angle $(80 \mathrm{mrad})$, the visibility of the $\mathrm{O}$ sublattice in the original map is partly due to elastic contrast (elastic scattering makes the $\mathrm{Ba}$ and $\mathrm{Sr}$ columns darker which effectively enhances the $\mathrm{O}$ sublattice). As with all corrected maps, the corrected $O-K$ map is expected to have purer chemical contrast. For the Ti- $L_{2,3}$ map, the correction does not produce an obvious change in contrast because, even though the Ti-O column intensity is enhanced, the intensity off the Ti-O columns (i.e., on the Ba and $\mathrm{Sr}$ columns) increases even more after correction.

Simulations enable analysis of the approximations underlying the correction of elastic contrast. To this end, atomicresolution elemental maps of $\mathrm{BaTiO}_{3}$, with its strongly scattering $\mathrm{Ba}$, were simulated using a multislice theory that incorporates scattering due to core-level excitations. ${ }^{19}$ Simulations included multiple-elastic, multiple-thermaldiffuse (via frozen-phonon algorithm), and single-core-loss scattering. Elastic and thermal-diffuse scattering both before and after each core-loss events was included. The simulations employed a supercell consisting of $8 \times 8$ projected unit cells of [001] $\mathrm{BaTiO}_{3}$ sampled using $512 \times 512$ pixels, and were performed using a GPU-based $\operatorname{code}^{23}$ which was run on a GPU cluster.

Figure 2(a) shows simulations of conventional and elastic-corrected elemental maps of [001] $\mathrm{BaTiO}_{3}$, where the 

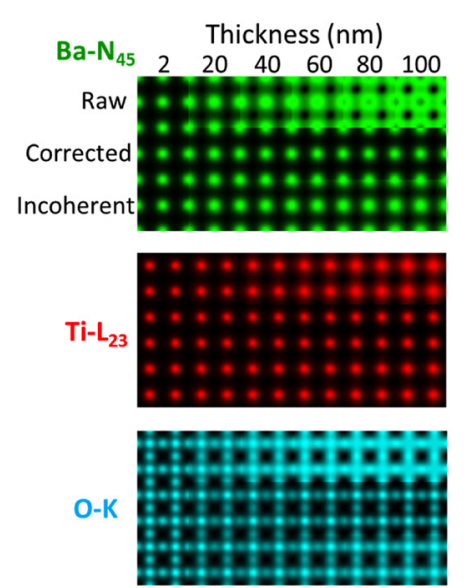

$\mathrm{Ba}-\mathrm{M}_{4}$

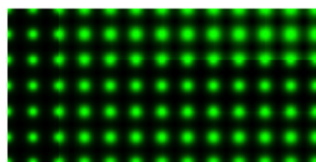

(a)

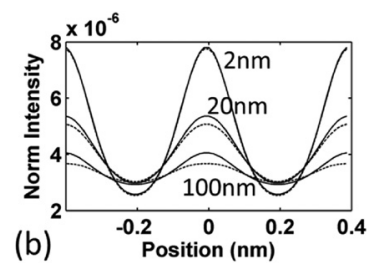

(b)

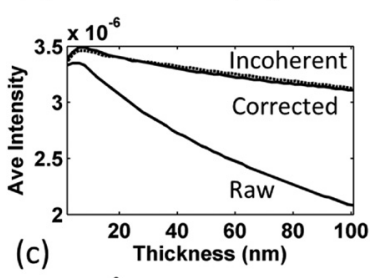

(C)

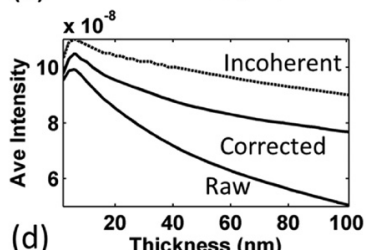

(d)

Thickness $(\mathrm{nm})$

FIG. 2. Theoretical analysis of elastic correction in atomic-resolution elemental mapping of [001] $\mathrm{BaTiO}_{3}$ as a function of sample thickness. (a) Simulated raw, corrected, and incoherent elemental maps; (b) simulated thickness-normalized line profiles across $\mathrm{Ba}$ atomic columns in corrected (solid lines) and incoherent (dotted lines) Ba- $N_{4,5}$ maps; (c) simulated thickness-normalized average $\mathrm{Ba}-N_{4,5}$ intensities; (d) as in (c) but for $\mathrm{Ba}-M_{4,5}$.

probe, detector, and source size parameters match the experimental data in Fig. 1. From Fig. 2(a), it is seen that for thin samples, where elastic scattering is minimal, the conventional maps are directly interpretable with strong intensity peaks coinciding with the relevant atomic columns. In thicker samples, where elastic scattering can be very strong, the conventional $\mathrm{Ba}-M_{4,5}$ and $\mathrm{Ti}-L_{2,3}$ maps exhibit significantly broader peaks, while the Ba- $N_{4,5}$ and O- $K$ maps exhibit nonintuitive behaviour with local minima on some or all of the relevant atomic columns. In particular, the Ba- $N_{4,5}$ map, whose relatively low energy loss entails significant inelastic delocalization, is dramatically influenced by the strong elastic scattering caused by $\mathrm{Ba}$. The corrected maps, on the other hand, remain intuitively interpretable at least up to $100 \mathrm{~nm}$, with narrow peaks coinciding with the relevant atomic columns. The effect of correction is especially noticeable for the $\mathrm{Ba}-N_{4,5}$ and $\mathrm{O}-K$ maps. These findings correlate very well with the experimental results in Fig. 1.

Also shown in Fig. 2(a) are simulated incoherent elemental maps, whereby the entire core-loss signal at a given energy-loss is collected, i.e., as for characteristic x-ray maps, there is no collection cutoff. Hence such maps are highly amendable to a direct interpretation, and they can be regarded as the ideal result against which the elastic-corrected maps should be compared. To enable this comparison on a quantitative level, Fig. 2(b) shows a thickness-normalized line profile across $\mathrm{Ba}$ columns in the $\mathrm{Ba}-N_{4,5}$ map. It is seen that the corrected and incoherent line profiles agree very well, with the largest discrepancy being about $10 \%$ at $100 \mathrm{~nm}$ thickness. Similar agreement is obtained for the other core-loss signals (not shown). As a further comparison, Figs. 2(c) and 2(d) show thickness-normalized average intensities in the Ba- $N_{4,5}$ and $\mathrm{Ba}-M_{4,5}$ maps. For the lower-loss $\mathrm{Ba}-N_{4,5}$ case the

corrected and incoherent results are again in excellent agreement, while for the higher-loss $\mathrm{Ba}-M_{4,5}$ case the discrepancy reaches about $10 \%$ at $100 \mathrm{~nm}$ thickness. This trend is attributed to the deviation of inelastic scattering characteristics from elastic scattering, which is expected to be greater at higher losses. As a general rule, the corrected maps exhibit slightly narrower peaks than the incoherent ones, as is discernible from the maps in Fig. 2(a). This behaviour indicates a slight over-correction, the details of which will be published elsewhere.

It should be noted that, while the corrected maps exhibit purer chemical contrast, effects due to probe scattering before core-loss excitations can still be present after correction, just as they are for characteristic x-ray maps and ADF images. Hence to achieve a fully quantitative interpretation of the map intensity and contrast, such effects will need to be taken into account.

We further applied the elastic correction to experimental low-loss maps of the heterostructure in Fig. 3. Figure 3(b) shows a spectrum image obtained by averaging the 2D lowloss map over the vertical dimension following Fourier-log deconvolution to remove multiple-scattering effects. The spectrum image exhibits peaks at 27.5 and $30 \mathrm{eV}$, corresponding to bulk plasmons in $\mathrm{BaTiO}_{3}{ }^{24}$ and $\mathrm{SrTiO}_{3}{ }^{25}$ respectively. The plasmon peaks are most clearly seen in the averaged spectra from the center of each layer (Fig. 3(c)). The shoulder on the $\mathrm{SrTiO}_{3}$ plasmon peak seems to arise from the $\mathrm{BaTiO}_{3}$ plasmon, which is plausible given the relevant inelastic delocalization is a few Angstroms. ${ }^{26}$ Low-loss maps used integration windows encompassing the $\mathrm{BaTiO}_{3}$ and $\mathrm{SrTiO}_{3}$ plasmon peaks, shifted slightly from the peak centers to maximize the layer contrast. The resulting maps in Figs. 3(d) and 3(e) exhibit not only the layer contrast but also strong atomic-resolution elastic contrast, with strong intensity minima on heavy-atom columns. This elastic contrast is essentially completely eliminated in the corrected maps in Figs. 3(f) and 3(g), verifying that the IBF image (Fig. 1(b)) and the low-loss maps have the same elastic contrast, and hence validating our approach.
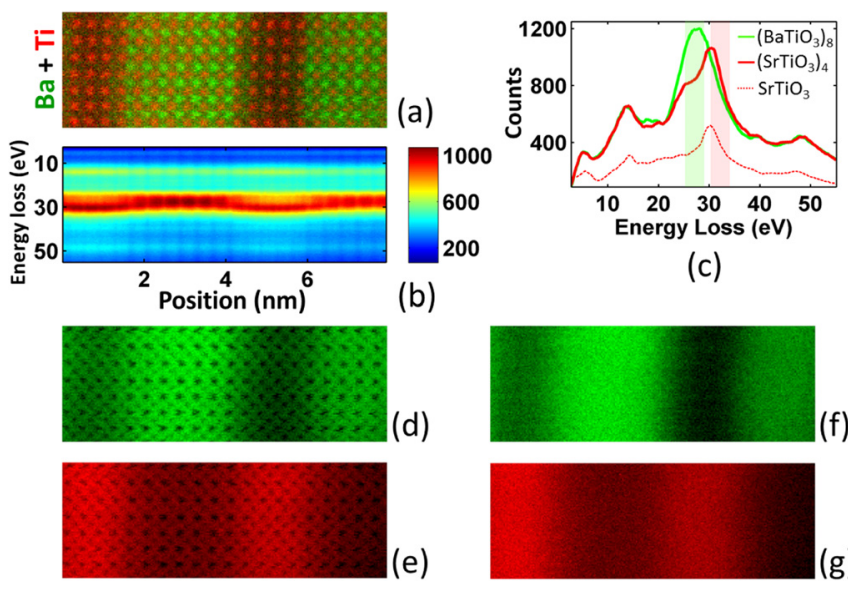

(c)

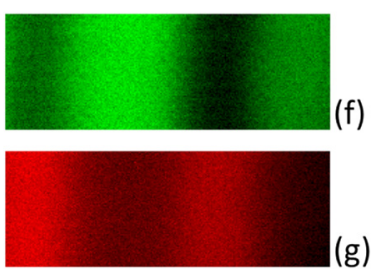

FIG. 3. Plasmon mapping of the $\left(\mathrm{BaTiO}_{3}\right)_{8} /\left(\mathrm{SrTiO}_{3}\right)_{4}$ heterostructure. (a) Composite of Ba- $M_{4,5}$ and Ti- $L_{2,3}$ maps; (b) $1 \mathrm{D}$ low-loss spectrum image; (c) low-loss spectra from $\left(\mathrm{BaTiO}_{3}\right)_{8}$ and $\left(\mathrm{SrTiO}_{3}\right)_{4}$ layers showing integration windows selecting plasmon peaks, along with $\mathrm{SrTiO}_{3}$ reference spectrum (dotted line); (d, e) 2D plasmon maps; (f, g) elastic-corrected plasmon maps. 
In summary, we have shown that the simultaneouslyacquired elastic signal can be used to correct elasticscattering artifacts in atomic-resolution STEM-EELS elemental maps. The corrected maps exhibit purer chemical contrast, making them more likely to be intuitively interpretable in terms of the relevant underlying sublattice. The correction can also be applied to remove atomic-scale elastic contrast in low-loss mapping techniques, such as plasmon mapping. Finally, it is noted that a simultaneously-acquired ADF image could also be used to generate the elasticcorrection map provided that the ADF intensity is properly normalized, the inner ADF and EELS collection angles coincide, and scattering beyond the outer ADF angle can be neglected. On the other hand, the approach used here is both more direct and entails the benefits of recording the low-loss spectra, including the ability to perform mapping based on low-energy excitations.

The work at Monash was supported by the Australian Research Council (DP110104734). The work at Cornell was supported by the U.S. Department of Energy, Office of Basic Energy Sciences, Division of Materials Sciences and Engineering (DE-SC0002334).

${ }^{1}$ E. Okunishi, H. Sawada, Y. Kondo, and M. Kersker, Microsc. Microanal. 12(Supp 2), 1150 (2006).

${ }^{2}$ M. Bosman, V. J. Keast, J. L. García-Muñoz, A. J. D’Alfonso, S. D. Findlay, and L. J. Allen, Phys. Rev. Lett. 99, 086102 (2007).

${ }^{3}$ K. Kimoto, T. Asaka, T. Nagai, M. Saito, Y. Matsui, and K. Ishizuka, Nature 450, 702 (2007).

${ }^{4}$ D. A. Muller, L. Fitting Kourkoutis, M. Murfitt, J. H. Song, H. Y. Hwang, J. Silcox, N. Dellby, and O. L. Krivanek, Science 319, 1073 (2008).

${ }^{5}$ G. A. Botton, S. Lazar, and C. Dwyer, Ultramicroscopy 110, 926 (2010).
${ }^{6}$ L. Fitting Kourkoutis, H. L. Xin, T. Higuchi, Y. Hotta, J. H. Lee, Y. Hikita, D. G. Schlom, H. Y. Hwangand, and D. A. Muller, Philos. Mag. 90, 4731 (2010).

${ }^{7}$ M. Chu, S. C. Liou, C. Chang, F. Choa, and C. H. Chen, Phys. Rev. Lett. 104, 196101 (2010).

${ }^{8}$ A. J. D'Alfonso, B. Freitag, D. Klenov, and L. J. Allen, Phys. Rev. B 81, 100101 (2010).

${ }^{9}$ L. Gunawan, S. Lazar, O. Gautreau, C. Harnagea, A. Pignolet, and G. A. Botton, Appl. Phys. Lett. 95, 192902 (2009).

${ }^{10}$ H. Tan, S. Turner, E. Yücelen, J. Verbeeck, and G. Van Tendeloo, Phys. Rev. Lett. 107, 107602 (2011).

${ }^{11}$ J. A. Mundy, Q. Mao, C. M. Brooks, D. G. Schlom, and D. A. Muller, Appl. Phys. Lett. 101, 042907 (2012).

${ }^{12}$ M. P. Oxley, M. Varela, T. J. Pennycook, K. Van Benthem, S. D. Findlay, A. J. D'Alfonso, L. J. Allen, and S. J. Pennycook, Phys. Rev. B 76, 064303 (2007).

${ }^{13}$ A. J. D'Alfonso, S. D. Findlay, M. P. Oxley, and L. J. Allen, Ultramicroscopy 108, 677 (2008).

${ }^{14}$ C. Dwyer, S. D. Findlay, and L. J. Allen, Phys. Rev. B 77, 184107 (2008).

${ }^{15}$ A. Soukiassian, W. Tian, V. Vaithyanathan, J. H. Haeni, L. Q. Chen, X. X. Xi, D. G. Schlom, D. A. Tenne, H. P. Sun, X. Q. Pan, et al., J. Mat. Res. 23, 1417 (2008).

${ }^{16}$ K. Kathan-Galipeau, P. P. Wu, Y. L. Li, L. Q. Chen, A. Soukiassian, Y. Zhu, D. A. Muller, X. X. Xi, D. G. Schlom, and D. A. Bonnell, J. Appl. Phys. 112, 052011 (2012).

${ }^{17}$ P. Cueva, R. Hovden, J. A. Mundy, H. L. Xin, and D. A. Muller, Microsc. Microanal. 18, 667 (2012).

${ }^{18}$ H. Kohl and H. Rose, Adv. Electronic Electron. Phys. 65, 173 (1985).

${ }^{19}$ C. Dwyer, Ultramicroscopy 104, 141 (2005).

${ }^{20}$ P. Wang, A. D'Alfonso, S. Findlay, L. Allen, and A. Bleloch, Phys. Rev. Lett. 101, 236102 (2008).

${ }^{21}$ E. C. Cosgriff, M. P. Oxley, L. J. Allen, and S. J. Pennycook, Ultramicroscopy 102, 317 (2005).

${ }^{22}$ C. Dwyer, H. Xin, and D. Muller, Phys. Rev. B 86, 094119 (2012).

${ }^{23}$ C. Dwyer, Ultramicroscopy 110, 195 (2010).

${ }^{24} \mathrm{~V}$. Gallegos-Orozco, R. Martínez-Sánchez, and F. Espinosa-Magaña, Phys. Rev. B 77, 045128 (2008).

${ }^{25}$ T. Yamazaki, Y. Kotaka, M. Tsukada, and Y. Kataoka, Ultramicroscopy 110, 1161 (2010).

${ }^{26}$ D. A. Muller and J. Silcox, Ultramicroscopy 59, 195 (1995). 